\title{
Trigger factors for rupture of intracranial aneurysms in relation to patient and aneurysm characteristics
}

\author{
Monique H. M. Vlak • Gabriel J. E. Rinkel • \\ Paut Greebe · Johanna G. van der Bom • \\ Ale Algra
}

Received: 28 October 2011/Revised: 22 November 2011/Accepted: 24 November 2011/Published online: 21 December 2011

(C) The Author(s) 2011. This article is published with open access at Springerlink.com

\begin{abstract}
Female gender, age above 60 years, and an aneurysm larger than $5 \mathrm{~mm}$ or location on the posterior circulation are associated with a higher rupture risk of intracranial aneurysms. We hypothesized that this association is explained by a higher susceptibility to (one of) the eight trigger factors that were recently identified. We included 250 patients with aneurysmal subarachnoid hemorrhage. We calculated relative risks (RR) with $95 \%$ confidence intervals $(95 \% \mathrm{CI})$ of aneurysmal rupture for trigger factors according to sex, age, site, and size of the aneurysms by means of the case-crossover design. None of the triggers except for physical exercise differed according to patient and aneurysm characteristics. In the hour after exposure to physical exercise: (1) patients over the age of 60 have a six-times-higher risk of rupture (RR 13; 95\% CI $6.3-26)$ than those of 60 years of age and under (RR 2.3; $1.3-4.1)$; (2) aneurysms at the internal carotid artery have a higher risk than those at other locations (RR 17; 7.8-37), but this was only statistically significant when compared to
\end{abstract}

M. H. M. Vlak · G. J. E. Rinkel · P. Greebe · A. Algra Department of Neurology and Neurosurgery, Rudolf Magnus Institute of Neuroscience, Utrecht Stroke Center, University Medical Center Utrecht, Utrecht, The Netherlands

M. H. M. Vlak · A. Algra (

Julius Center for Health Sciences and Primary Care, University

Medical Center Utrecht, mailbox STR 6.131, PO Box 85500,

3508 GA Utrecht, The Netherlands

e-mail: a.algra@umcutrecht.nl

M. H. M. Vlak

Department of Neurology, Slotervaart Hospital,

Amsterdam, The Netherlands

J. G. van der Bom · A. Algra

Department of Clinical Epidemiology, Leiden University

Medical Center, Leiden, The Netherlands anterior communicating artery aneurysms (RR 3.2; 1.66.1); (3) aneurysms $5 \mathrm{~mm}$ or smaller had a higher risk of rupture (RR 9.5; 4.6-19) than larger aneurysms (RR 2.4; 1.3-4.3); and (4) women and men had similar risks. A higher susceptibility to exercise might explain part of the higher risk of rupture in older patients. Why women and patients with aneurysms larger than $5 \mathrm{~mm}$ or posterior circulation aneurysms have a higher risk of rupture remains to be settled.

Keywords Subarachnoid hemorrhage - Risk factors · Trigger factors $\cdot$ Intracranial aneurysm

\section{Introduction}

Three percent of the general population harbors an intracranial aneurysm and the number of incidentally discovered unruptured intracranial aneurysms is rising with the increasing quality and availability of non-invasive imaging techniques [1,2]. Only a minority of aneurysms rupture, but when this happens, it is usually a disabling or fatal occurrence [3].

The precise pathophysiological mechanism of aneurysmal rupture is not completely understood. It is important to identify risk factors for aneurysmal rupture in order to tailor treatment of intracranial aneurysms. Female gender and an age above 60 years are factors associated with an increased risk of rupture, as are an aneurysm size of more than $5 \mathrm{~mm}$ and location of the aneurysm on the posterior circulation [4]. However, the reason why the risk of rupture is higher in females, patients over 60 years of age, and patients with a large aneurysm or one located at the posterior circulation, is still poorly understood [4]. 
We recently identified eight trigger factors for aneurysmal rupture, namely coffee and cola consumption, physical exercise, sneezing, straining for defecation, sexual intercourse, anger, and being startled [5]. We hypothesized that a different susceptibility to trigger factors could explain the difference in risk of rupture between patients of different sex and age and between aneurysms of different sites and sizes. We therefore assessed the risk of aneurysmal rupture by eight confirmed trigger factors according to sex and age of the patients and according to site and size of the aneurysm.

\section{Methods}

Design and study population

The design of this study is described elsewhere [5]. In short, we performed a case-crossover study among patients who had been admitted to the Utrecht Stroke Center for aneurysmal SAH. Aneurysmal SAH was defined as an abrupt onset of severe headache or loss of consciousness, with or without focal neurological signs, with subarachnoid blood proven by neuro-imaging or lumbar puncture, and a radiologically proven aneurysm. The study protocol was approved by the Medico-Ethical Review Committee of our hospital.

\section{Procedures}

The case-crossover design enables studying the effect of transient exposure to potential trigger factors on the risk of an acute event, such as subarachnoid hemorrhage (SAH), by comparing exposure in a period shortly before the event with the patient's usual frequency of exposure [6]. In the present study, we included as determinants only those factors that were found to be trigger factors in our previous case-crossover study [5]. For these triggers, we had collected information on exposure in the past year, the usual frequency of exposure, and the presence of exposure in the "hazard period", which we defined as the hour before $\mathrm{SAH}$, according to the estimated duration of effect of each trigger.

For each patient, we collected data on sex and age, as well as on site and size of the ruptured aneurysm for each patient. Age was categorized as 60 years or younger or as above 60 years, because in a previous review risk of rupture increased above this age limit [4]. Site was categorized as follows: anterior cerebral artery, anterior communicating artery, and pericallosal artery combined (ACA), internal carotid artery, posterior communicating artery, ophthalmic artery, and anterior choroidal artery combined (ICA), middle cerebral artery (MCA), and vertebrobasilar arteries
(VBA). In addition, we also combined the ACA, ICA, and MCA into an anterior circulation (ANT) group. Sizes were categorized as $\leq 5 \mathrm{~mm}, 5.1-10 \mathrm{~mm}$ and $>10 \mathrm{~mm}$ based on the largest diameter of the aneurysm.

Data analysis

With the case-crossover design, exposure to potential trigger factors in the hour before onset of SAH (hazard period) was compared with the usual frequency over the year before the $\mathrm{SAH}$ and was expressed as relative risk (RR) with corresponding 95\% confidence intervals (CI) [7].

This analysis was done for men and women, patients over 60 years, and those of 60 years of age and under, and also for each site- and size-category. RRs with $95 \%$ CIs of different age-, gender-, site-, and size-categories were compared between categories to assess whether they overlapped. We also performed analyses with site- and size-categories combined.

\section{Results}

We included 250 patients (62 men and 188 women) with aneurysmal SAH. The mean age was 54.7 years (55.7 years for men, 54.3 years for women). Nineteen men and 63 women were over 60 years old.

Of the 250 ruptured aneurysms 150 (60.0\%) were $>5 \mathrm{~mm}$ and $24(9.6 \%)$ were $>10 \mathrm{~mm}$. Aneurysms were located in the ACA in $41.6 \%$, MCA $20.0 \%$, ICA $28.0 \%$ and in the VBA in $10.4 \%$ (Table 1 ).

\section{Sex}

Data on trigger factors according to sex are given in Table 2. We found no statistically significant difference between men and women for any of the trigger factors.

Table 1 Site and size of 250 ruptured aneurysms in 250 included patients

\begin{tabular}{lll}
\hline Location & \multicolumn{2}{l}{ Size } \\
\cline { 2 - 3 } & $\leq 5 \mathrm{~mm}(n=100)$ & $>5 \mathrm{~mm}(n=150)$ \\
\hline ACA & 52 & 52 \\
ICA & 26 & 44 \\
MCA & 17 & 33 \\
VBA & 5 & 21 \\
\hline
\end{tabular}

$A C A$ anterior communicating artery, anterior cerebral artery, and pericallosal artery combined, ICA internal carotid artery, posterior communicating artery, ophthalmic artery, and anterior choroidal artery combined, $M C A$ middle cerebral artery, $V B A$ vertebrobasilar arteries 
Table 2 Relative risks for eight trigger factors according to sex and age

\begin{tabular}{|c|c|c|c|c|}
\hline \multirow[t]{2}{*}{ Trigger factor } & \multicolumn{2}{|l|}{ Sex } & \multicolumn{2}{|l|}{ Age } \\
\hline & $\operatorname{Men}(n=62)$ & Women $(n=188)$ & $\leq 60$ years $(n=168)$ & $>60$ years $(n=82)$ \\
\hline Coffee & $2.2(1.3-3.8)$ & $2.7(1.9-3.9)$ & $2.5(1.7-3.6)$ & $2.6(1.5-4.5)$ \\
\hline Cola & $3.6(0.9-13.9)$ & $5.5(2.3-13)$ & $4.7(2.2-10)$ & $6.0(0.9-40)$ \\
\hline Nose blowing & $2.1(0.6-7.1)$ & $4.1(2.3-7.5)$ & $3.4(1.8-6.4)$ & $3.5(1.3-9.7)$ \\
\hline Straining for defecation & $7.4(1.8-30)$ & $15.3(6.1-39)$ & $8.9(3.3-24)$ & $23(6.7-79)$ \\
\hline Being startled & NP & $40(10-165)$ & $41(6.1-276)$ & $28(3.9-194)$ \\
\hline Anger & $14(3.5-56)$ & $22(6.6-76)$ & $20(7.2-56)$ & $13(1.8-103)$ \\
\hline Sexual intercourse & $28(11-71)$ & $10(3.9-25)$ & $17(8.7-34)$ & $7.7(1.03-56)$ \\
\hline Physical exercise & $1.9(0.9-4.2)$ & $6.2(3.6-11)$ & $2.3(1.3-4.1)$ & $13(6.3-26)$ \\
\hline
\end{tabular}

Values are RR $(95 \% \mathrm{CI})$

$R R$ Relative risks and corresponding 95\% confidence intervals, $N P$ No patients in this category, therefore no RR could be calculated, Exercise vigorous to extreme physical exercise (MET $>6$ )

Age

Data on trigger factors according to age are given in Table 2. Patients above 60 years have a significantly higher risk of rupture after physical exercise than patients of 60 years or younger. A similar pattern was observed after stratification for usual frequency of exercise into $<3$ vs. $\geq 3$ times per week. For all other trigger factors, there was no statistically significant difference.

Site

For all trigger factors, the RR was not statistically different between site categories, except for physical exercise (Table 3). The risk of rupture after exposure to physical exercise was higher for aneurysms located on the ICA compared to those on the ACA, but not with those on the
MCA. None of the ruptures of ICA aneurysms were triggered by being startled and none of the VBA aneurysms were triggered by physical exercise or anger. When relative risks of ANT aneurysms were compared to the VBA aneurysms $95 \%$ CI overlapped for all trigger factors.

\section{Size}

Initially, sizes were categorized as $\leq 5 \mathrm{~mm}, 5.1-10 \mathrm{~mm}$, and $>10 \mathrm{~mm}$ based on the largest diameter of the aneurysm. However, due to the small number of aneurysms $>10 \mathrm{~mm}$ in our study, the $95 \%$ CI overlapped with the other categories (data not shown). Therefore, we dichotomized size as $\leq 5 \mathrm{~mm}$ and $>5 \mathrm{~mm}$.

The risk of rupture in the hour after physical exercise was higher for aneurysms $\leq 5 \mathrm{~mm}$ compared to aneurysms $>5 \mathrm{~mm}$. For all other trigger factors, the risk of

Table 3 Relative risks for trigger factors according to aneurysm site

\begin{tabular}{|c|c|c|c|c|c|}
\hline \multirow[t]{2}{*}{ Trigger factor } & \multicolumn{5}{|c|}{ Site-ruptured aneurysm } \\
\hline & ACA $(n=104)$ & ICA $(n=70)$ & $\operatorname{MCA}(n=50)$ & ANT $(n=224)$ & $\operatorname{VBA}(n=26)$ \\
\hline Coffee & $2.7(1.7-4.2)$ & $3.9(2.0-7.6)$ & $1.7(0.9-3.3)$ & $2.6(1.9-3.6)$ & $1.9(0.8-4.5)$ \\
\hline Cola & $19(4.9-72)$ & $2.1(0.3-14)$ & $3.5(0.9-14)$ & $5.7(2.6-12.6)$ & $2.0(0.3-13.2)$ \\
\hline Nose blowing & $2.3(0.9-5.8)$ & $6.6(2.2-18)$ & $6.2(2.2-18)$ & $3.8(2.2-6.9)$ & $1.9(0.4-8.5)$ \\
\hline Straining for defecation & $13(4.6-39)$ & $6.5(0.8-54)$ & $8.6(0.9-87)$ & $11(4.5-26)$ & $23(5.3-98)$ \\
\hline Being startled & $40(1.5-1098)$ & NP & $46(6.3-339)$ & $29(5.0-173)$ & $57(8.1-406)$ \\
\hline Anger & $20(4.8-88)$ & $38(7.2-196)$ & $7.9(1.2-53)$ & $20(7.8-48)$ & NP \\
\hline Sexual intercourse & $20(7.5-52)$ & $11(2.5-48)$ & $4.7(0.7-34)$ & $12(5.9-25)$ & $47(11-203)$ \\
\hline Exercise & $3.2(1.6-6.1)$ & $17(7.8-37)$ & $3.2(0.8-12.8)$ & $5.1(3.3-8.1)$ & NP \\
\hline
\end{tabular}

Values are RR $(95 \% \mathrm{CI})$

$R R$ Relative risks and corresponding 95\% confidence intervals, $A C A$ anterior communicating artery, anterior cerebral artery, and pericallosal artery combined, ICA internal carotid artery, posterior communicating artery, ophthalmic artery, and anterior choroidal artery combined, $M C A$ middle cerebral artery, $A N T$ Anterior circulation (combining ACA, MCA and ICA), VBA vertebrobasilar arteries, NP No patients in this category, therefore no RR could be calculated, Exercise vigorous to extreme physical exercise $(\mathrm{MET}>6)$ 
Table 4 Relative risks for trigger factors according to aneurysm size

\begin{tabular}{lll}
\hline Trigger factor & \multicolumn{2}{l}{ Aneurysm size } \\
\cline { 2 - 3 } & $\leq 5 \mathrm{~mm}(n=100)$ & $>5 \mathrm{~mm}(n=150)$ \\
\hline Coffee & $2.2(1.3-3.9)$ & $2.7(1.9-4.0)$ \\
Cola & $9.8(2.4-41)$ & $3.7(1.6-8.8)$ \\
Nose blowing & $3.6(1.4-9.0)$ & $3.4(1.8-6.5)$ \\
Straining for defecation & $20(7.5-52)$ & $5.4(1.3-22)$ \\
Being startled & $59(7.3-479)$ & $20(2.8-139)$ \\
Anger & $9.0(1.3-63)$ & $23.8(8.3-69)$ \\
Sexual intercourse & $15(5.0-45)$ & $15.7(7.1-35)$ \\
Exercise & $9.5(4.6-19)$ & $2.4(1.3-4.3)$ \\
\hline
\end{tabular}

Values are RR $(95 \% \mathrm{CI})$

Small, aneurysms size $\leq 5 \mathrm{~mm}$; Large, aneurysm size $>5 \mathrm{~mm}$

$R R$ Relative risks and corresponding 95\% confidence intervals, Exercise vigorous to extreme physical exercise (MET $>6$ )

rupture did not statistically significantly differ between size categories (Table 4). We did an additional analysis for ACA aneurysms of $\leq 5 \mathrm{~mm}$ and $>5 \mathrm{~mm}$, since ACA was the most common site of aneurysms. The RR of ACA aneurysms $\leq 5 \mathrm{~mm}$ was $17(7.2-39)$, but $0.5(0.1-2.9)$ for ACA aneurysms $>5 \mathrm{~mm}$.

\section{Discussion}

We found that patients over the age of 60 have a higher risk of aneurysmal rupture in the hour after exposure to physical exercise than patients under the age of 60 . No difference in trigger factors was found between women and men. We also did not find an increased susceptibility for trigger factors for aneurysms located at the posterior circulation or for aneurysms $>5 \mathrm{~mm}$ in size. In contrast, our results suggest a higher risk of rupture for aneurysms $\leq 5 \mathrm{~mm}$ after exposure to physical exercise, in particular when located at the ACA.

A review on risk factors for rupture of intracranial aneurysms showed a higher risk for women and patients above the age of 60 [4]. We found a higher risk of rupture after physical exercise for patients above 60 , which may partly explain the higher rupture rate in older patients. When interpreting the risk increase of exercise among the elderly, one should realize nevertheless that they exercise less frequently than younger persons. There are few studies on cerebrovascular response to exercise in elderly people [8-10]. One of these studies reported a delay in cerebral autoregulation in older persons compared to younger persons $[9,10]$. Since exercise causes a rise in systemic blood pressure, a delayed cerebral autoregulation may lead to an initial short-lasting increase in mean cerebral blood flow velocity. However, not all studies agree on differences in cerebral autoregulation between younger and older persons $[8,9]$, therefore our hypothesis remains a speculative one.

The risk of rupture of ICA aneurysms after exposure to vigorous physical exercise seems higher compared to that of ACA aneurysms. Possibly, the arterial blood pressure is transmitted more directly to the most proximal cerebral vessels, such as the ICA and BA, which in turn may lead to a higher rupture rate for aneurysms at the ICA and BA. However, no data are available comparing blood flow in different cerebral vessels during exercise.

We found a higher risk of rupture after exposure to physical exercise for aneurysms sized $\leq 5 \mathrm{~mm}$ compared to aneurysms $>5 \mathrm{~mm}$, which was mainly due to a very high risk for small ACA aneurysms. Others have already suggested that ACA aneurysms might be unstable and rupture at a small size, while those that reach a larger size are a selection of more stable aneurysms [11, 12]. Our findings support this theory and may partly explain the high number of small ACA aneurysms in SAH studies, while ACA aneurysms are less prevalent in studies on unruptured aneurysms [4]. However, since our analysis on size and site combined was an additional analysis on a smaller group of aneurysms, this may also be a chance finding. Only two earlier reports have been published on trigger factors for SAH, but they did not differentiate according to site and size $[13,14]$. Hence, we cannot further compare our results with those in the literature.

The present study includes information on trigger factors from 250 patients, which may be considered as a strong point given the severity of the illness. The use of the case-crossover design (no control selection bias) and the inclusion of patient-derived data only (increasing reliability and completeness of data) are other strengths of our study. However, the present study also has some limitations. Of the 250 included patients, only $10 \%$ had an aneurysm in the VBA, which makes it difficult (and for anger and physical exercise even impossible) to compare rupture risks with other sites. Another limitation is that multiple testing and subgroup analyses on smaller numbers of aneurysms may have led to chance findings.

If our data on higher susceptibility to physical exercise as a trigger factor for older patients and patients with small ACA aneurysms are confirmed by others, the next step would be to further study the hemodynamics in intracranial vessels after exercise in these subgroups. Furthermore, trigger factors should be studied in Finnish and Japanese patients, since aneurysms in these populations have a higher risk of rupture [4]. This might reveal a different susceptibility to trigger factors per site and size and could even yield new trigger factors. The higher rupture rates in women and the predisposition for rupture of aneurysms $>5 \mathrm{~mm}$ and aneurysms located at the posterior 
circulation can so far not be explained by a difference in susceptibility to trigger factors and remain to be unraveled.

Acknowledgments This study was funded by the Julius Center for General Health and Primary Care and the Department of Neurology and Neurosurgery of the University Medical Center Utrecht.

\section{Conflicts of interest None.}

Open Access This article is distributed under the terms of the Creative Commons Attribution Noncommercial License which permits any noncommercial use, distribution, and reproduction in any medium, provided the original author(s) and source are credited.

\section{References}

1. Vlak MH, Algra A, Brandenburg R, Rinkel GJ (2011) Prevalence of unruptured intracranial aneurysms, with emphasis on sex, age, comorbidity, country, and time period: a systematic review and meta-analysis. Lancet Neurol 10:626-636

2. Gabriel RA, Kim H, Sidney S, McCulloch CE, Singh V, Johnston SC et al (2010) Ten-year detection rate of brain arteriovenous malformations in a large, multiethnic, defined population. Stroke 41:21-26

3. Nieuwkamp DJ, Setz LE, Algra A, Linn FH, de Rooij NK, Rinkel GJ (2009) Changes in case fatality of aneurysmal subarachnoid haemorrhage over time, according to age, sex, and region: a meta-analysis. Lancet Neurol 8:635-642

4. Wermer MJ, van der Schaaf I, Algra A, Rinkel GJ (2007) Risk of rupture of unruptured intracranial aneurysms in relation to patient and aneurysm characteristics: an updated meta-analysis. Stroke 38:1404-1410

5. Vlak MH, Rinkel GJ, Greebe P, van der Bom JG, Algra A (2011) Trigger factors and their attributable risk for rupture of intracranial aneurysms: a case-crossover study. Stroke 42:1878-1882
6. Maclure M (1991) The case-crossover design: a method for studying transient effects on the risk of acute events. Am J Epidemiol 133:144-153

7. Mittleman MA, Maclure M, Sherwood JB, Mulry RP, Tofler GH, Jacobs SC et al (1995) Triggering of acute myocardial infarction onset by episodes of anger. Determinants of myocardial infarction onset study investigators. Circulation 92:1720-1725

8. Fisher JP, Ogoh S, Young CN, Raven PB, Fadel PJ (2008) Regulation of middle cerebral artery blood velocity during dynamic exercise in humans: influence of aging. J Appl Physiol 105:266-273

9. Heckmann JG, Brown CM, Cheregi M, Hilz MJ, Neundorfer B (2003) Delayed cerebrovascular autoregulatory response to ergometer exercise in normotensive elderly humans. Cerebrovasc Dis 16:423-429

10. Ogoh S, Fisher JP, Young CN, Fadel PJ (2011) Impact of age on critical closing pressure of the cerebral circulation during dynamic exercise in humans. Exp Physiol 96:417-425

11. Wiebers DO, Whisnant JP, Sundt TM Jr, O'Fallon WM (1987) The significance of unruptured intracranial saccular aneurysms. J Neurosurg 66:23-29

12. Wiebers DO, Piepgras DG, Meyer FB, Kallmes DF, Meissner I, Atkinson JL et al (2004) Pathogenesis, natural history, and treatment of unruptured intracranial aneurysms. Mayo Clin Proc 79:1572-1583

13. Anderson C, Ni MC, Scott D, Bennett D, Jamrozik K, Hankey G (2003) Triggers of subarachnoid hemorrhage: role of physical exertion, smoking, and alcohol in the Australasian Cooperative Research on subarachnoid hemorrhage study (ACROSS). Stroke 34:1771-1776

14. Fann JR, Kukull WA, Katon WJ, Longstreth WT Jr (2000) Physical activity and subarachnoid haemorrhage: a population based case-control study. J Neurol Neurosurg Psychiatry 69:768772 